\title{
The Number of Generalized Balanced Lines
}

\author{
David Orden · Pedro Ramos • Gelasio Salazar
}

Received: 22 May 2009 / Revised: 1 February 2010 / Accepted: 7 February 2010 /

Published online: 2 March 2010

(C) Springer Science+Business Media, LLC 2010

\begin{abstract}
Let $S$ be a set of $r$ red points and $b=r+2 \delta$ blue points in general position in the plane, with $\delta \geq 0$. A line $\ell$ determined by them is balanced if in each open half-plane bounded by $\ell$ the difference between the number of blue points and red points is $\delta$. We show that every set $S$ as above has at least $r$ balanced lines. The proof is a refinement of the ideas and techniques of Pach and Pinchasi (Discrete Comput. Geom. 25:611-628, 2001), where the result for $\delta=0$ was proven, and introduces a new technique: sliding rotations.
\end{abstract}

Keywords Balanced partitions · Halving triangles · Generalized Lower Bound Theorem · Circular sequences · Allowable sequences $\cdot$ Sliding rotations

\section{Introduction}

Let $B$ and $R$ be, respectively, sets of blue and red points in the plane, and let $S=B \cup R$ be in general position. Let $r=|R|$ and $b=|B|=r+2 \delta$ with $\delta \geq 0$. Furthermore, we are given weights $\omega(p)=+1$ for $p \in B$ and $\omega(q)=-1$ for $q \in R$.

This work was started at the 6th Iberian Workshop on Computational Geometry, in Aveiro, and was concluded while Gelasio Salazar was visiting Department of Mathematics of Alcalá University under the program Giner de los Ríos.

D. Orden and P. Ramos are partially supported by grants MTM2008-04699-C03-02 and HP2008-0060.

D. Orden · P. Ramos $(\bowtie)$

Departamento de Matemáticas, Universidad de Alcalá, Alcalá de Henares, Spain e-mail: pedro.ramos@uah.es

D. Orden

e-mail: david.orden@uah.es

G. Salazar

Instituto de Física, Universidad Autónoma de San Luis Potosí, San Luis Potosí, Mexico e-mail: gsalazar@dec1.ifisica.uaslp.mx 
Given a halfplane $H$, its weight is then defined as $\omega(H)=\sum_{s \in S \cap H} \omega(s)$. Here and throughout this paper, halfplanes are open unless otherwise stated.

Definition 1 A line $\ell$ determined by two points of $S$ is balanced if the two halfplanes it defines have weight $\delta$. Observe that this implies that the two points of $S$ spanning $\ell$ have different colors.

The main result of this paper is an elementary, geometric proof for the following lower bound on the number of balanced lines.

Theorem 1 Let $B$ and $R$ be, respectively, sets of blue and red points in the plane, and let $S=B \cup R$ be in general position. Let $r=|R|$ and $b=|B|=r+2 \delta$ with $\delta \geq 0$. The number of lines defined by two points of $S$ that divide the plane in two halfplanes of weight $\delta$ is at least $r$. This number is attained if $R$ and $B$ can be separated by $a$ line.

For $\delta=0$, we obtain the result conjectured by George Baloglou and proved by Pach and Pinchasi via circular sequences:

Theorem 2 ([3]) Let $|R|=|B|=n$. Every set $S$ as above determines at least $n$ balanced lines. This bound is tight.

The result for $\delta>0$ was proved by Sharir and Welzl [4] in an indirect manner, via an equivalence with the following very special case of the Generalized Lower Bound Theorem. This motivated them to ask for a more direct and simpler proof.

Let $\mathcal{P}$ be a convex polytope which is the intersection of $d+4$ halfspaces in general position in $\mathbb{R}^{d}$. Let its edges be oriented according to a generic linear function (edges are directed from smaller to larger value; "generic" means that the function evaluates to distinct values at the vertices of $\mathcal{P}$ ).

Theorem 3 ([4]) The number of vertices with $\left\lceil\frac{d}{2}\right\rceil-1$ outgoing edges is at most the number of vertices with $\left\lceil\frac{d}{2}\right\rceil$ outgoing edges.

Finally, let us remark that in [4] it is also shown that Theorem 1 is equivalent to the following result about halving triangles:

Theorem 4 Every set $S \subset \mathbb{R}^{3}$ of $2 n+1$ points in general position has at least $n^{2}$ halving triangles.

All proofs in this paper can be easily translated to the more general setting of circular sequences (see [2]).

\section{Geometric Tools}

We assume that coordinate axes are chosen in such a way that all points have different abscissa. The tools we use are inspired in the rotational movement introduced by Erdős et al. [1]. 
Fig. 1 Transitions in an $R^{k}$-rotation are always through a balanced line

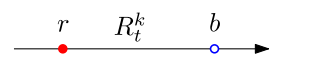

$\delta \rightsquigarrow \delta+1$

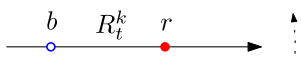

$\delta+1 \rightsquigarrow \delta$

Definition 2 Let $P \subseteq S$. A $P^{k}$-rotation is a family of directed lines $P_{t}^{k}$, where $t \in$ $[0,2 \pi]$ is the angle measured from the vertical axis, defined as follows: $P_{0}^{k}$ contains a single point of $P$, and as $t$ increases, it rotates counterclockwise in such a way that

(i) $\left|P \cap P_{t}^{k}\right|=1$ except for a finite number of events when $\left|P \cap P_{t}^{k}\right|=2$.

(ii) Whenever $\left|P \cap P_{t}^{k}\right|=1$, there are exactly $k$ points of $P$ to the right of $P_{t}^{k}$.

The common point $P \cap P_{t}^{k}=\{p\}$ is called the pivot, and it changes precisely when $\left|P \cap P_{t}^{k}\right|=2$. Observe that $P_{0}^{k}=P_{2 \pi}^{k}$.

Definition 3 Let $\ell^{+}$and $\ell^{-}$denote, respectively, the open halfplanes to the right and to the left of $\ell$. Let $\omega(\ell)$ be the weight of $\ell^{+}$. Given a $P^{k}$-rotation, we say that $P^{k} \geq \delta$ if $\omega\left(P_{t}^{k}\right) \geq \delta$ for every $t \in[0,2 \pi]$, and similarly for the rest of inequalities. A rotation $B^{k}$ is $\delta$-preserving if either $B^{k} \geq \delta$ or $B^{k}<\delta$. Symmetrically, $R^{k}$ is $\delta$-preserving if either $R^{k} \leq \delta$ or $R^{k}>\delta$.

Lemma 5 In an $R^{k}$-rotation, transitions $\delta \rightsquigarrow \delta+1$ and $\delta+1 \rightsquigarrow \delta$ in $\omega\left(R_{t}^{k}\right)$ are always through a balanced line. In a $B^{k}$-rotation, transitions $\delta \rightsquigarrow \delta-1$ and $\delta-1 \rightsquigarrow \delta$ in $\omega\left(B_{t}^{k}\right)$ are always through a balanced line.

Proof When a red point is found during an $R^{k}$-rotation, the weight of the halfplane is preserved because the pivot point changes. Therefore, the change $\delta \rightsquigarrow \delta+1$ happens when a blue point is found in the head of $R_{t}^{k}$ (Fig. 1, left), while $\delta+1 \rightsquigarrow \delta$ happens when a blue point is found in the tail of $R_{t}^{k}$ (Fig. 1, right). In both cases, the points define a balanced line. For a $B^{k}$-rotation, the proof is identical.

Claim 8.1 in [3] has now a more direct proof:

Lemma 6 If $r$ is odd, there exists a balanced line which is a halving line of $S$.

Proof Let $k=\left\lfloor\frac{r}{2}\right\rfloor$ and consider an $R^{k}$-rotation. If $R_{0}^{k} \leq \delta$, then $R_{\pi}^{k}>\delta$, and conversely. Therefore, there exist transitions $\delta \rightsquigarrow \delta+1$ and $\delta+1 \rightsquigarrow \delta$ in $\omega\left(R_{t}^{k}\right)$ which, by Lemma 5 , are always through a balanced line. Observe that both transitions are through the same balanced line, with angles $t_{0}$ and $t_{0}+\pi$.

Remark 1 Let us observe that Theorem 1.4 in [3], which states that Theorem 2 is true when $R$ and $B$ are separated by a line $\ell$, has now an easier proof: if we start $R^{k}$-rotations with a line parallel to $\ell$, for each $k$, there exist exactly one transition $\delta \rightsquigarrow \delta+1$ and one transition $\delta+1 \rightsquigarrow \delta$ which, by Lemma 5, correspond always to a balanced line. If $r$ is even, there are two balanced lines for $k=0, \ldots, \frac{r}{2}-1$, for a total of $r$ balanced lines, while if $r$ is odd, there are two balanced lines for $k=0, \ldots,\left\lfloor\frac{r}{2}\right\rfloor-1$ and one balanced line for $k=\left\lfloor\frac{r}{2}\right\rfloor$. 
Remark 2 Lemmas 5 and 6 conclude the proof of Theorem 2 if no $R^{k}$-rotation is $\delta$ preserving or if no $B^{k}$-rotation (with $k \geq \delta$ ) is $\delta$-preserving. Hence, in the following we assume that there exists either at least one $R^{k}$-rotation or one $B^{k}$-rotation (with $k \geq \delta$ ) which is $\delta$-preserving.

Lemma 7 Let $0 \leq j \leq\left\lfloor\frac{r}{2}\right\rfloor$. If $R^{j}>\delta$, then $B^{j+\delta} \geq \delta$, while if $B^{j+\delta}<\delta$, then $R^{j} \leq \delta$.

Proof Consider the line $R_{t_{0}}^{j}$. The halfplane $\left(R_{t_{0}}^{j}\right)^{+}$contains $j$ red points and $b>j+\delta$ blue points. Therefore, the line $B_{t_{0}}^{j+\delta}$ is to the right of $\left(R_{t_{0}}^{j}\right)^{+}$and contains at most $j$ red points. Then, $\omega\left(B_{t_{0}}^{j+\delta}\right) \geq \delta$. The proof of the second statement is analogous.

The next definition generalizes the concept of $P^{k}$-rotation in two different ways: parallel movements are permitted, and the number of points to the right of the line can change.

Definition 4 A $P$-sliding rotation consists in moving a directed line $\ell$ continuously, starting with an $\ell_{0}$ which contains a single point $p_{0} \in P$, and composing rotation around a point of $P$ (the pivot) and parallel displacement (in either direction) until the next point of $P$ is found. Furthermore, after a $2 \pi$ rotation is completed, the line $\ell_{0}$ must be reached again.

This movement is clearly a continuous curve in the space of lines in the plane. For instance, if a line is parameterized as a point in $S^{1} \times \mathbb{R}$, a $P$-sliding rotation describes a (nonstrictly) angular-wise monotone curve, with vertical segments corresponding to parallel displacements.

Let $\Sigma$ be a $P$-sliding rotation. Let us denote by $\Sigma_{t}$ the line with angle $t$ with respect to the vertical axis defined as follows: if there is no parallel displacement at angle $t$, then $\Sigma_{t}$ denotes the corresponding line. Otherwise, it denotes the leftmost line corresponding to angle $t$.

Definition 5 A $P$-sliding rotation $\Sigma$ is simple if $\Sigma_{t+\pi}$ is to the left of $\Sigma_{t}$ for all $t \in$ $[0, \pi)$.

The inequality $\Sigma \geq \delta$, as well as the remaining inequalities, is defined exactly as in Definition 3. Similarly, a $B$-sliding rotation $\Sigma$ is $\delta$-preserving if $\Sigma \geq \delta$, while an $R$-sliding rotation is $\delta$-preserving if $\Sigma \leq \delta$. The following definition is the crux of the rest of the paper.

Definition 6 Let $\Delta$ be the set of all simple, $\delta$-preserving $B$-sliding rotations and $R$-sliding rotations. The waist of a $P$-sliding rotation $\Sigma \in \Delta$ is

$$
\min _{t \in[0, \pi]}\left|P \cap \Sigma_{t}^{-} \cap \Sigma_{t+\pi}^{-}\right| .
$$

We denote by $\Gamma$ the sliding rotation of $\Delta$ with the smallest waist. 
Note that the set $\Delta$ is nonempty because we have assumed that there exist $\delta$ preserving $B^{k}$ - or $R^{k}$-rotations, which are a particular type of sliding rotations. Furthermore, the waist takes only a finite number of values, so it has a minimum. If the minimum is not unique, we can pick any of the sliding rotations achieving it.

\section{Main Result}

Assume that $\Gamma$ is a $\delta$-preserving $R$-sliding rotation (i.e., $\Gamma \leq \delta$ ). In this case, we will manage to prove that there exist at least $r$ balanced lines. For the case of $\Gamma$ being a $\delta$-preserving $B$-sliding rotation, the same arguments would show that there exist at least $b$ balanced lines.

Lemma 8 Let $\Gamma_{0}$ and $\Gamma_{\pi}$ be the lines achieving the waist of $\Gamma$, let $\bar{\Gamma}_{0}^{+}$be the closed halfplane to the right of $\Gamma_{0}$, and let $F=R \cap \bar{\Gamma}_{0}^{+}$. For every $k \in\{0, \ldots,|F|-1\}$, during an $F^{k}$-rotation, a balanced line is found. Similarly, let $H=R \cap \bar{\Gamma}_{\pi}^{+}$. For every $k \in\{0, \ldots,|H|-1\}$, during an $H^{k}$-rotation, a balanced line is found.

Proof Figure 2 illustrates the situation. On the one hand, $F_{0}^{k}$ is to the right of $\Gamma_{0}$, and, since $\Gamma$ is simple, $F_{\pi}^{k}$ is to the left of $\Gamma_{\pi}$. This implies that there is a $t_{1} \in[0, \pi]$ such that $F_{t_{1}}^{k}=\Gamma_{t_{1}}$, and therefore $\omega\left(F_{t_{1}}^{k}\right) \leq \delta$. On the other hand, $F_{0}^{k}$ is to the left of $\Gamma_{\pi}$, and $F_{\pi}^{k}$ is to the right of $\Gamma_{0}$; therefore, there exists a $t_{2} \in[0, \pi]$ such that $F_{t_{2}}^{k}$ and $\Gamma_{t_{2}+\pi}$ are the same line with opposite directions. Since $\omega\left(\Gamma_{t_{2}+\pi}\right) \leq \delta$, then $\omega\left(F_{t_{2}}^{k}\right) \geq \delta$. If $\omega\left(\Gamma_{t_{2}+\pi}\right)=\delta$ and the line contains a blue point, then it is a balanced line found in a transition $\delta \rightsquigarrow \delta+1$. Otherwise, $\omega\left(F_{t_{2}}^{k}\right)>\delta$, and hence a transition $\delta \rightsquigarrow \delta+1$ has occurred for a $t \in\left(t_{1}, t_{2}\right)$.

Now, observe that $R \backslash F \subset \Gamma_{0}^{-}$. Hence, in the $F^{k}$-rotation for $t \in[0, \pi]$, all the points in $R \backslash F$ are found by the head of the line. This implies that a change $\delta \rightsquigarrow \delta+1$ in the weight of the right halfplane can only occur when a blue point is found in the head of the ray (as in Fig. 1, left), hence defining a balanced line. The proof for $H$ is identical.

Observe that balanced lines found in this process are different, because they have exactly $k$ points of $F$, respectively $H$, to the right.

Let now $C_{t}^{\Gamma}$ be the central region of the sliding rotation $\Gamma$ at instant $t$ defined as $C_{t}^{\Gamma}=\Gamma_{t}^{-} \cap \Gamma_{t+\pi}^{-}$. Observe that, for the corresponding $t$, the transitions $\delta \rightsquigarrow \delta+1$ in the proof of Lemma 8 correspond to balanced lines inside or in the boundary of the central region.

Fig. 2 Illustration of the proof of Lemma 8

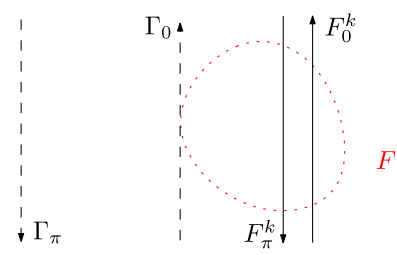


Lemma 9 Let $G=R \backslash(F \cup H)$. For $k \in\{0, \ldots,\lceil|G| / 2\rceil-1\}$, every $G^{k}$-rotation has transitions $\delta \rightsquigarrow \delta+1$ and $\delta+1 \rightsquigarrow \delta$, which correspond to lines inside or in the boundary of the central region., i.e., for the corresponding $t, G_{t}^{k} \in C_{t}^{\Gamma}$.

Proof Let us consider first the case where $r$ is odd and $k=\lfloor|G| / 2\rfloor . G_{0}^{k}$ and $G_{\pi}^{k}$ are the same line with opposite directions. Therefore, if $\omega\left(G_{0}^{k}\right) \leq \delta$, then $\omega\left(G_{\pi}^{k}\right)>\delta$, and there must be at least two transitions as stated. These transitions correspond to lines in the central region because $\Gamma$ is simple.

For the remaining cases, observe that, by construction, $G_{0}^{k} \in C_{0}^{\Gamma}$. According to the value of $\omega\left(G_{0}^{k}\right)$, we distinguish two cases:

- $\omega\left(G_{0}^{k}\right) \leq \delta$. If there exist some values for which $G_{t}^{k}=\Gamma_{t}$, let $t_{1}$ and $t_{2}$ be, respectively, the minimum and maximum of them. If there is no such value, take $t_{1}=t_{2}=2 \pi$. If $G^{k}$ takes the value $\delta+1$ in the interval $\left(0, t_{1}\right)$, it must have transitions $\delta \rightsquigarrow \delta+1$ and $\delta+1 \rightsquigarrow \delta$, and the same is true for $\left(t_{2}, 2 \pi\right)$. Finally, observe that $G^{k}$ must take the value $\delta+1$ at least once, because in the other case the sliding rotation obtained by concatenating $G^{k}$ in $\left(0, t_{1}\right), \Gamma$ in $\left(t_{1}, t_{2}\right)$, and $G^{k}$ in $\left(t_{2}, 2 \pi\right)$ would be a $\delta$-preserving sliding rotation of waist smaller than the waist of $\Gamma$.

- $\omega\left(G_{0}^{k}\right)>\delta$. If there exist some values for which $G_{t}^{k}=\Gamma_{t}$, let $t_{1}$ and $t_{2}$ be, respectively, the minimum and maximum of them. $G_{t}^{k}$ takes the value $\delta$ in the intervals $\left(0, t_{1}\right)$ and $\left(t_{2}, 2 \pi\right)$, and therefore the lemma follows. In other case, if $G_{t}^{k}$ takes the value $\delta$ in the central region, it must have also transition $\delta \rightsquigarrow \delta+1$. Finally, if $\omega\left(G_{t}^{k}\right)>\delta$ for all $t \in[0,2 \pi]$, we could construct a sliding rotation $\Sigma$ contradicting the choice of $\Gamma$ : for each $t$, consider as $\Sigma_{t}$ the parallel to $G_{t}^{k}$ which passes through the first blue point to the right of $G_{t}^{k}$. It is easy to see that $\Sigma_{t} \geq \delta$, because between $\Gamma_{t}$ and $G_{t}^{k}$ there are always at least two blue points.

The following lemma, which already appeared as Claim 6.4 in [3], will be enough to conclude the proof of Theorem 2 .

Lemma 10 Transitions $\delta \rightsquigarrow \delta+1$ and $\delta+1 \rightsquigarrow \delta$ in a $G^{k}$-rotation are always either a balanced line or a $\delta+1 \rightsquigarrow \delta$ transition in an $F^{j}$-rotation, $j \in\{0, \ldots,|F|-1\}$, or an $H^{j}$-rotation, $j \in\{0, \ldots,|H|-1\}$.

Proof On the one hand, a balanced line is achieved if there is such a transition because a blue point is found. See Fig. 1 . On the other hand, if the point inducing the transition is $r \in R$, then necessarily $r \in R \backslash G$ (since the $G^{k}$-rotation changes pivot whenever a point of $G$ is found). Figure 3 illustrates that a $\delta+1 \rightsquigarrow \delta$ transition appears for an $F^{j}$-rotation with pivot $g$, both if $f \in F$ is found in the tail (left picture) or if $f \in F$ is found in the head (right picture). Note that in the right picture the

Fig. 3 Transitions when a point $f \in F \subset R$ found in a $G^{k}$-rotation induces a $\delta+1 \rightsquigarrow \delta$ transition in an $F^{j}$-rotation. In both cases, the pivot is $g$

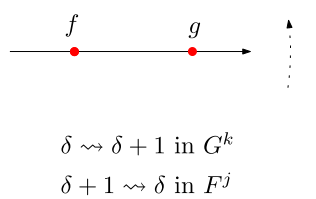

$\delta+1 \rightsquigarrow \delta$ in $F^{j}$

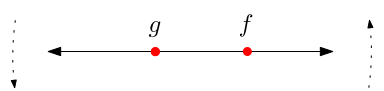

$\delta+1 \rightsquigarrow \delta$ in $G^{k}$

$\delta+1 \rightsquigarrow \delta$ in $F^{j}$ 
weight of both halfplanes is $\delta+1$. The case in which the point found is $h \in H$ works similarly.

\section{Proof of Theorem 1}

(a) Lemma 8 gives $|F|+|H|$ different balanced lines.

(b) Lemmas 9 and 10 give $|G|$ lines which are either a balanced line or a $\delta+1 \rightsquigarrow \delta$ transition at the central region for an $F^{j}$ - or $H^{j}$-rotation.

(c) Each $\delta+1 \rightsquigarrow \delta$ transition in (b) forces a new $\delta \rightsquigarrow \delta+1$ transition at the central region for an $F^{j}$ - or $H^{j}$-rotation which correspond, as in the proof of Lemma 8, to a new balanced line.

Acknowledgements The authors thank Jesús García for helpful discussions.

\section{References}

1. Erdős, P., Lovász, L., Simmons, A., Strauss, E.G.: Dissection graphs on planar point sets. In: A Survey of Combinatorial Theory, pp. 139-149. North Holland, Amsterdam (1973)

2. Orden, D., Ramos, P., Salazar, G.: Balanced lines in two-coloured point sets. arXiv:0905.3380v1 [math.CO]

3. Pach, J., Pinchasi, R.: On the number of balanced lines. Discrete Comput. Geom. 25, 611-628 (2001)

4. Sharir, M., Welzl, E.: Balanced lines, halving triangles, and the generalized lower bound theorem. In: Aronov, B., Basu, S., Pach, J., Sharir, M. (eds.) Discrete and Computational Geometry-The Goodman-Pollack Festschrift, pp. 789-798. Springer, Heidelberg (2003) 